\title{
Hydrothermal Unzipping of Multiwalled Carbon Nanotubes and Cutting of Graphene by Potassium Superoxide
}

\author{
Apostolos Koutsioukis ${ }^{1}$, Konstantinos Spyrou ${ }^{2} \mathbb{D}$, Nikolaos Chalmpes ${ }^{2} \mathbb{D}$, Dimitrios Gournis ${ }^{2, *(\mathbb{D})}$ \\ and Vasilios Georgakilas $1, * \mathbb{D}$ \\ 1 Department of Materials Science, University of Patras, 26504 Rio, Greece; up1057091@upatras.gr \\ 2 Department of Materials Science and Engineering, University of Ioannina, 45110 Ioannina, Greece; \\ konstantinos.spyrou1@gmail.com (K.S.); chalmpesnikos@gmail.com (N.C.) \\ * Correspondence: dgourni@uoi.gr (D.G.); viegeorgaki@upatras.gr (V.G.)
}

Citation: Koutsioukis, A.; Spyrou, K.; Chalmpes, N.; Gournis, D.; Georgakilas, V. Hydrothermal Unzipping of Multiwalled Carbon Nanotubes and Cutting of Graphene by Potassium Superoxide. Nanomaterials 2022, 12, 447. https:// doi.org/10.3390/nano12030447

Academic Editor: Filippo Giannazzo

Received: 23 December 2021

Accepted: 27 January 2022

Published: 28 January 2022

Publisher's Note: MDPI stays neutral with regard to jurisdictional claims in published maps and institutional affiliations.

Copyright: (C) 2022 by the authors. Licensee MDPI, Basel, Switzerland. This article is an open access article distributed under the terms and conditions of the Creative Commons Attribution (CC BY) license (https:// creativecommons.org/licenses/by/ $4.0 /)$.

\begin{abstract}
The dual use of potassium superoxide $\left(\mathrm{KO}_{2}\right)$ to unzip multiwalled carbon nanotubes (MWCNTs) and cut graphene under hydrothermal conditions is described in this work. The $\mathrm{KO}_{2-}$ assisted hydrothermal treatment was proven to be a high-yield method for forming graphene nanoribbons and dots or sub-micro-sized graphene nanosheets. Starting with functionalized MWCNTs, the method produces water-dispersible graphene nanoribbons with characteristic photoluminescence depending on their width. Using pristine graphene, the hydrothermal treatment with $\mathrm{KO}_{2}$ produces nanosized graphene sheets and graphene quantum dots with diameters of less than $10 \mathrm{~nm}$. The latter showed a bright white photoluminescence. The effective hydrothermal unzipping of MWNTs and the cutting of large graphene nanosheets is a valuable top-down approach for the preparation of graphene nanoribbons and small nanographenes. Both products with limited dimensions have interesting applications in nanoelectronics and bionanotechnology.
\end{abstract}

Keywords: graphene nanoribbons; graphene dots; nanographene; unzipping; hydrothermal treatment; potassium superoxide

\section{Introduction}

Graphene and carbon nanotubes (CNTs) are the two most well-studied carbon nano allotropes [1]. They are both structured with a hexagonal carbon lattice, which is extended in two dimensions in graphene and wrapped cylindrically in CNTs. Graphene nanosheets and CNTs have been widely studied the last decades in several applications that are related to their properties, such as electrical and thermal conductivity, optical transparency, chemical reactivity, and mechanical strength [2-5]. Thanks to their nanosized thickness, they have been often used as substrates in bioapplications and nanoelectronics [6-12]. One of the most challenging demands with regard to their application is the control of their size and dimensions. Therefore, their electrical and optical properties depend, among other things, on the size and the thickness of the graphene or CNTs. Bioapplications, such as drug or gene delivery, usually also need much smaller graphene nanosheets than those usually isolated from graphite $[13,14]$. On the other hand, a new graphenic nanostructure has been developed over the last few years called graphene nanoribbons (GNRs), and it is considered to be an attractive material, since it combines planar graphenic structures, including graphene nanosheets, and CNTs with narrow widths and very high aspect ratios [15]. Their electrical properties are strongly related to their width. Narrow GNRs appear to have a nonzero bandgap and can be used in field-effect transistors, while broad GNRs are conductive like graphene. Several studies have appeared in the literature describing their applications in quantum electronics, and in bionanotechnology with GNRs [15-18].

GNRs can be made, among other ways, by the unzipping of CNTs, which means making a cut in the longitudinal direction. Usually, their width ranges between 10 and 
$100 \mathrm{~nm}$, and their lengths are related to that of the starting CNTs [19-21]. The oxidative treatments of CNTs with a sulfuric acid solution of potassium permanganate $[19,20,22]$, potassium vapors [19], and palladium chloride ions $\left(\mathrm{PdCl}_{4}{ }^{-2}\right)$ [23] are characteristic procedures for the top-down approaches of nanoribbon formation. Several researchers have proposed, as a mechanism, the formation of manganate ester in lines, which is followed by the dissociation of carbon-carbon bonds through an oxidative or a reductive reaction [19]. Bottom-up methods have been also developed, combining aromatic molecules and specific organic reactions. In this way, narrow nanoribbons are constructed with widths of less than $10 \mathrm{~nm}$ [24-27].

Similar cutting methods have been also developed recently for graphene, leading to small-sized graphenic pieces with interesting optical properties: the so-called graphene quantum dots (GQDs) [28-30]. Their size ranges between 2 and $10 \mathrm{~nm}$ and they appear to exhibit a characteristic fluorescence emission upon excitation. Although the cutting of graphene or CNTs leads to different nanostructures, the proposed mechanism of cutting the common hexagonal carbon lattice is similar in both cases [31,32]. Hydrothermal treatment is a relatively new, very promising procedure that has been often used with graphene and CNTs and their derivatives. The limitation of water dispersibility is a disadvantage for the use of pristine graphene or carbon nanotubes; however, graphene oxide (GO), which is easily dispersible in water, is very often used [29,31-33]. Hydrothermal heating with potassium superoxide $\left(\mathrm{KO}_{2}\right)$ has been proven to be an effective method to produce GQDs from GO [34].

In this article, we explore the ability of $\mathrm{KO}_{2}$ to unzip functionalized water-dispersible multiwalled carbon nanotubes (MWCNTs) under hydrothermal conditions to create GNRs. Furthermore, we show that the same reaction is highly effective, as well, at cutting pristine graphene nanosheets to produce nanographenes and GQDs. The method is simple and eco-friendly, and can be performed at a large scale with good yield.

\section{Materials and Methods}

Potassium superoxide purchased from Sigma-Aldrich, MWNT-f-OH, were prepared according to [35]. Graphene was purchased from XG Science.

\subsection{Instrumentation}

FTIR spectra were obtained with an ATR technique on a Fourier transform spectrometer (IRTracer-100, Shimadzu Europa GmbH, Duisburg, Germany). Raman spectra were collected both with a Raman System T-64000 and a LabRam-HR Mirco Raman spectrometer (Horiba, NJ, USA) using a laser excitation line at $514 \mathrm{~nm}$. The laser power was $1.082 \mathrm{mV}$. XRD was conducted with a D8 Advance Bruker diffractometer (Bruker AXS, Karlsruhe, Germany) using a CuKa (ID 1.5418) radiation source ( $40 \mathrm{kV}, 40 \mathrm{~mA})$ and a secondary beam graphite monochromator. Diffraction patterns were recorded in the 2-theta $\left(2^{\prime}\right)$ scale from $2^{\circ}$ to $80^{\circ}$, in steps of $0.02^{\circ}$ and with a counting time of $2 \mathrm{~s}$ per step. The optical absorption spectra were obtained with a Hitachi Digilab, Model U2800-Double Beam-UV/Vis (Tokyo, Japan).

Surface roughness was measured by using AFM atomic force microscopy (AFM) images that were collected in tapping mode with a Bruker Multimode 3D Nanoscope (Ted Pella Inc., Redding, CA, USA) using a microfabricated silicon cantilever type TAP-300G, with a tip radius of $<10 \mathrm{~nm}$ and a force constant of approximately $20-75 \mathrm{~N} \mathrm{~m}^{-1}$. The Si wafers (P/Bor, single-side polished, Si-Mat) used in the AFM imaging were cleaned before use for $20 \mathrm{~min}$ in an ultrasonic bath $(160 \mathrm{~W})$ with water, acetone ( $\geq 99.5 \%$ Sigma-Aldrich, St. Louis, MO, USA), and ethanol ( $\geq 99.5 \%$ Sigma-Aldrich, St. Louis, MO, USA).

$X$-ray photoelectron spectroscopy (XPS) measurements were performed in an ultrahigh vacuum at a base pressure of $6 \times 10^{-9}$ mbar with a SPECS GmbH spectrometer equipped with a monochromatic $\mathrm{Mg} \mathrm{K} \alpha$ source $(\mathrm{hv}=1253.6 \mathrm{eV})$ and a Phoibos-100 hemispherical analyzer (Berlin, Germany). Spectral analysis included a Shirley background subtraction and peak separation using Gaussian-Lorentzian functions in a least squares 
fitting software package (Winspec) developed at the LISE laboratory, University of Namur, Belgium.

The sheet resistance of the samples was measured on circular spots made from the samples after being compressed at 2 tn by a 4-point probe system (Pro4 Resistivity System, Lucas Labs, Gilroy, CA, USA) and Keithley 2400 Source Meter. The thickness was estimated to be $5 \mu \mathrm{m}[35]$.

\subsection{Sample Preparation}

For this step, $5 \mathrm{mg}$ of catechol-functionalized MWCNTs [35] or pure graphene (XG Science) were dispersed in $6 \mathrm{~mL} \mathrm{H}_{2} \mathrm{O}$ by sonication for $30^{\prime}$, and then $50 \mathrm{mg}$ of $\mathrm{KO}_{2}$ was added, followed by stirring for another $30^{\prime}$. After that, the mixture was heated hydrothermally overnight at $185^{\circ} \mathrm{C}$ in an autoclave Teflon.

Carbon nanotubes: The product of the hydrothermal reaction was separated in two parts by precipitation: the supernatant $\left(\mathrm{NR}_{1}\right)$ and the precipitate $\left(\mathrm{NR}_{2}\right)$. The brown-toyellow-colored supernatant was purified with dialysis membrane, while the precipitate was washed and isolated by centrifugation $\left(15,000 \mathrm{rpm} / 15^{\prime}\right)$ and redispersed in water. The supernatant was left for a week on a filtration membrane to remove by-products.

Graphene nanosheets: The unreacted material or large graphene nanosheets were separated by precipitation. The liquid phase was left overnight and the precipitate that formed was separated by centrifugation $\left(15,000 \mathrm{rpm} / 15^{\prime}\right)(\mathrm{NG})$. The final brown-to-yellow supernatant was purified with a dialysis membrane (GQDs).

\section{Results}

\subsection{Unzipping of MWNTs}

Functionalized MWCNTs (MWCNT-f-OH) dispersed in water were mixed with an excess of $\mathrm{KO}_{2}$ in water and heated hydrothermally overnight at $185^{\circ} \mathrm{C}$. The mixture of the hydrothermal heating after sedimentation was separated in two parts: the supernatant $\left(\mathrm{NR}_{1}\right)$ and the precipitate $\left(\mathrm{NR}_{2}\right)$ (see Figure 1a). The brown-to-yellow-colored supernatant was purified using a dialysis membrane, while the precipitate was washed several times with water and isolated by centrifugation. The first evidence that indicated that different products were dispersed in the separated parts, apart from the color, was the photoluminescence of the $\mathrm{NR}_{1}$ sample, as shown in Figure $1 \mathrm{~b}$, upon excitation with a violet laser lamp (405 nm). The sample $\mathrm{NR}_{2}$ (diluted in water) showed no fluorescence under the same excitation (Figure 1c).

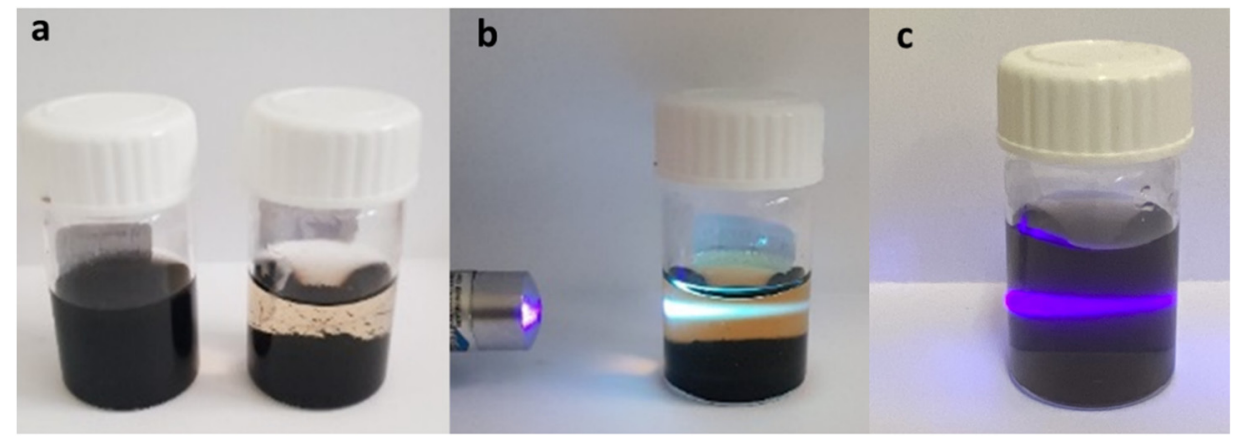

Figure 1. (a) MWCNT-f-OH in water before (left) and after (right) the hydrothermal treatment. $(\mathbf{b}, \mathbf{c})$ Excitation with violet laser lamp $(405 \mathrm{~nm})$ of the supernatant sample $\mathrm{NR}_{1}$ showing blue emission (b), and the precipitate sample $\mathrm{NR}_{2}$ redispersed in water (c) showing no emission. The violet light is due to the Tyndall effect of the dispersed $\mathrm{NR}_{2}$.

AFM images. The AFM images of the sample $\mathrm{NR}_{1}$ reveal that it contains elongated single-layered nanoribbons with a thickness of $0.86 \mathrm{~nm}$ or some thicker few layers nanoribbons ( $4 \mathrm{~nm})$ and a mean width close to 40 (see Figures $2 \mathrm{a}$,b and S1). The lower thickness indicates the formation of the thinnest one-layer nanoribbons. The precipitate sample, $\mathrm{NR}_{2}$, 
was also dispersible in water, creating a gray-to-black-colored dispersion, and it also contains elongated nanoribbons, with a larger width between 100 and $150 \mathrm{~nm}$ and a thickness between 2 and $3 \mathrm{~nm}$, as revealed by the AFM images (see Figures 2c and S2). Considering that a nanoribbon is formed by the unzipping of a MWCNT, it can be reasonably supposed that, here, the starting MWCNTs had an external layer with diameter between 30 and $50 \mathrm{~nm}$ that was unzipped, leading to the larger GNRs of sample $\mathrm{NR}_{2}$, while the internal layers that formed the narrower GNRs of sample $\mathrm{NR}_{1}$ behaved similarly (see Figure 3). The fact that the $\mathrm{NR}_{2}$ samples were less dispersible than the starting MWCNT-f-OH and precipitate in the reaction mixture (see Figure 1a) could be attributed to their different structure. MWCNT-f-OH is cylindrical, and the external surface is enriched by hydrophilic catechol groups that remarkably improve their dispersibility in water (see Figure 3). On the other hand, $\mathrm{NR}_{2}$ are plain structures with two hydrophobic aromatic surfaces, where only a part of them is decorated with catechols. The lower hydrophilic character of $\mathrm{NR}_{2}$ is due to the oxygen groups that formed at the edges after the hydrothermal scissoring.

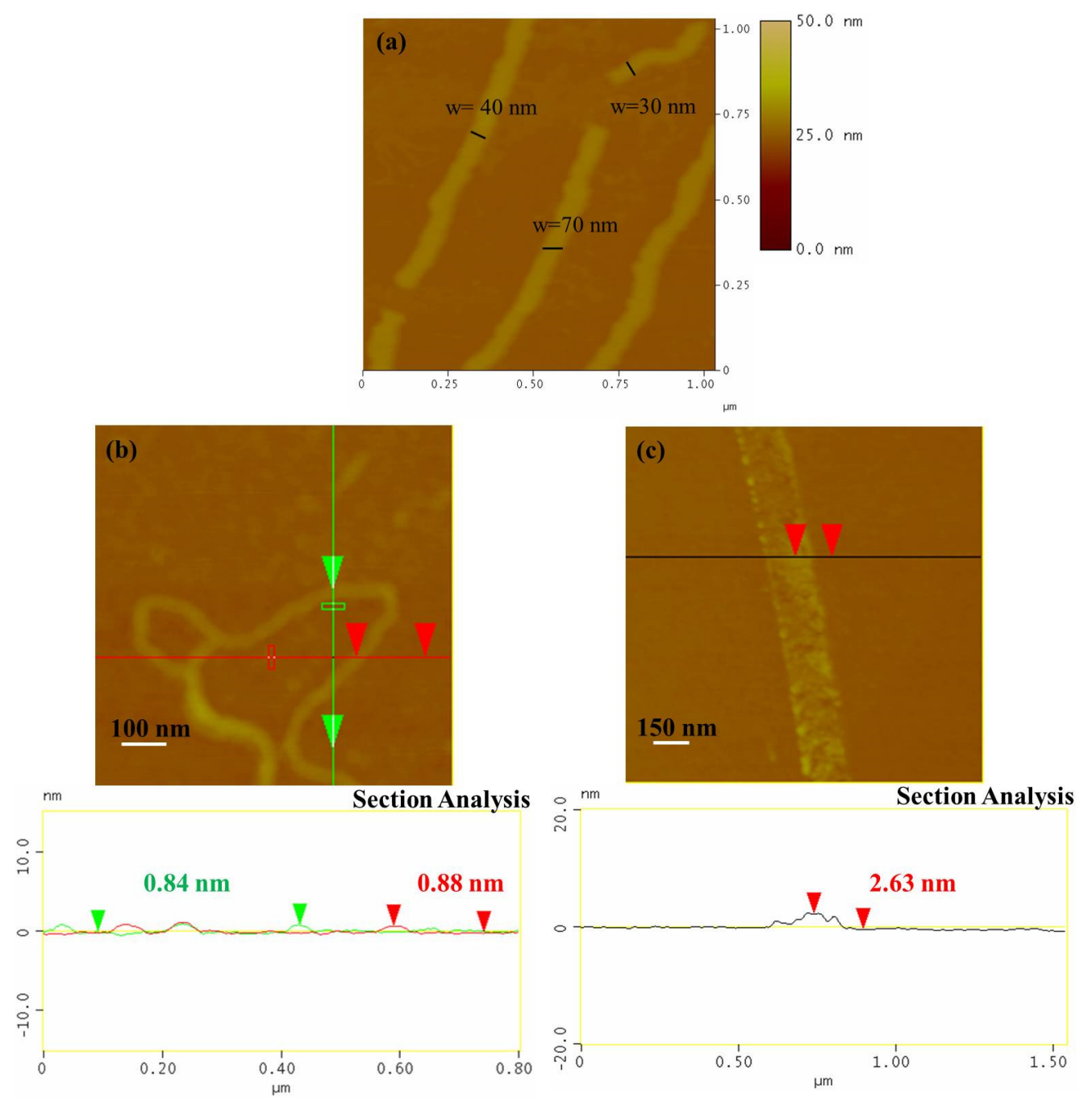

Figure 2. AFM images from the sample $\mathrm{NR}_{1}(\mathbf{a})$ and the sample $\mathrm{NR}_{2}(\mathbf{b}, \mathbf{c})$.

Photoluminescence. The photoluminescence (PL) of the samples $\mathrm{NR}_{1}$ and $\mathrm{NR}_{2}$ was evaluated using PL spectroscopy, as shown in Figure 4. The UV-Vis spectra of samples $\mathrm{NR}_{1}$ and $\mathrm{NR}_{2}$ were similar and show a broad absorption with a maximum at $263 \mathrm{~nm}$, which corresponds to the $\pi, \pi^{*}$ transition and a very broad, and weak absorption around $350 \mathrm{~nm}$ that corresponds to the $\mathrm{n}, \pi^{*}$ transition (see Figure 4a). A similar absorption curve was recorded by the MWCNT-f-OH sample. Although both $\mathrm{NR}_{1}$ and $\mathrm{NR}_{2}$ samples have similar UV-Vis absorption curves, only the sample $\mathrm{NR}_{1}$ showed photoluminescence. 
Under excitation with 250 to $450 \mathrm{~nm}$, sample $\mathrm{NR}_{1}$ showed an excitation that depended on luminescence, with the maximum ranged between 430 and $500 \mathrm{~nm}$. The most intense luminescence band has a $\lambda_{\max }$ at $446 \mathrm{~nm}$ under excitation with $350 \mathrm{~nm}$. The sample $\mathrm{NR}_{2}$ showed no photoluminescence, nor did the starting MWCNT-f-OHs.

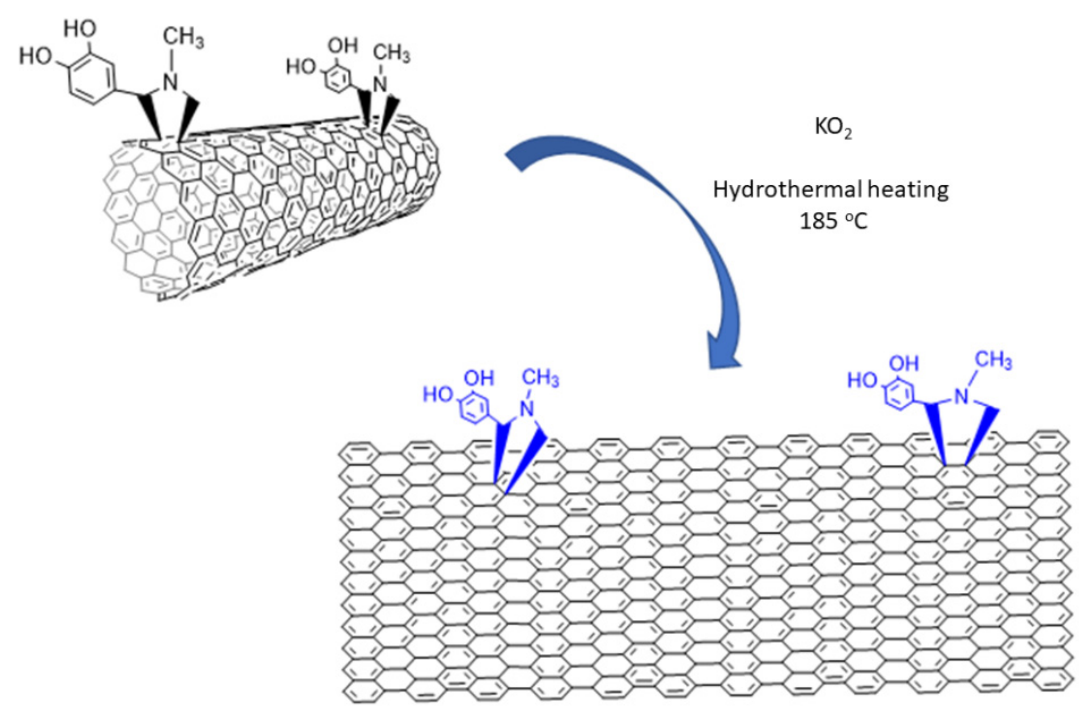

Figure 3. Unzipping of MWCNT-f-OH by $\mathrm{KO}_{2}$.
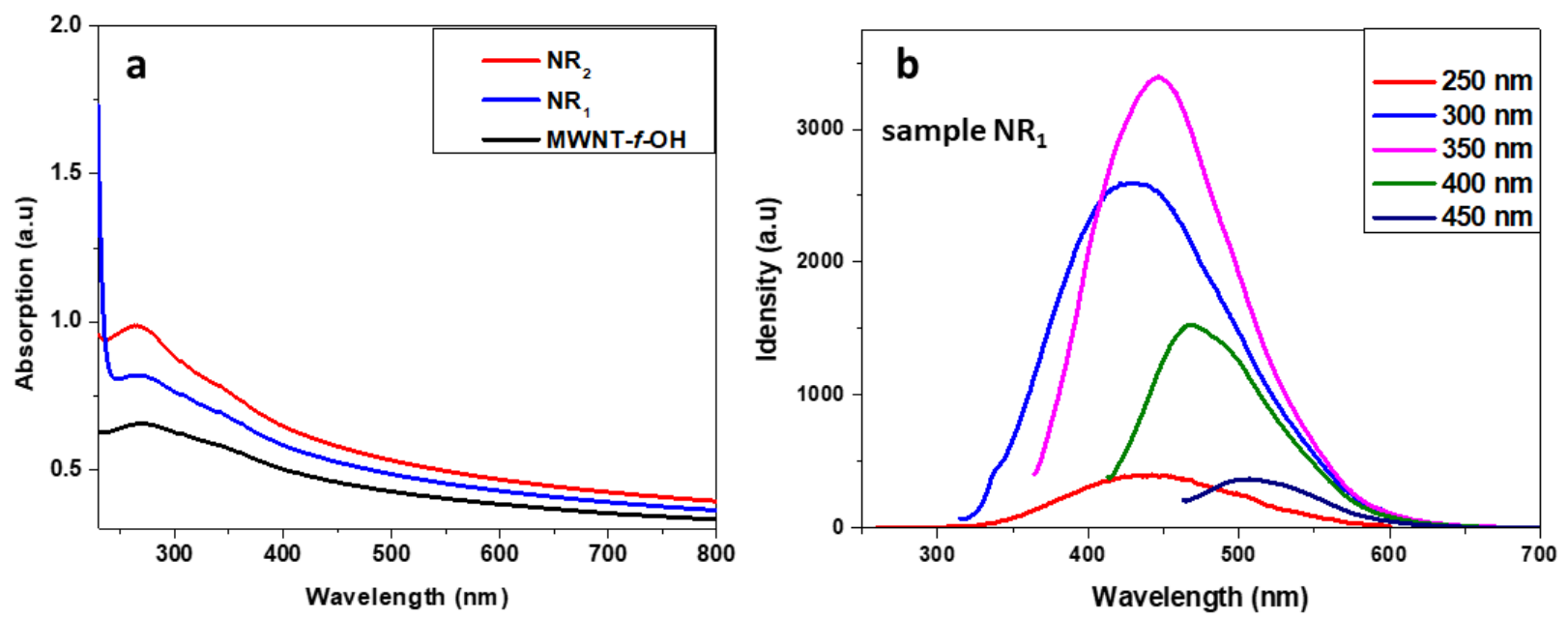

Figure 4. (a) UV-Vis spectra of samples $\mathrm{NR}_{1}, \mathrm{NR}_{2}$, and MWCNT-f-OH; (b) PL spectra of sample $\mathrm{NR}_{1}$ at several excitation wavelengths.

Considering the AFM images, the main difference between the two samples was the width of the nanoribbons. Hence, it is reasonably supposed that photoluminescence was determined by the width of the carbon nanoribbons [25]. In the literature, nanoribbons that have been detected with photoemission were mostly produced by bottom-up methods and their width was limited to $10 \mathrm{~nm}$ [24-27].

XRD and Raman. The XRD pattern of the MWCNT-f-OHs showed a characteristic peak at $26^{\circ}$ that corresponds to the $0.34 \mathrm{~nm}$ interlayer distance, which is characteristic between the graphenic walls of MWCNTs (see Figure 5). A similar peak did not appear in the XRD pattern of the product $\mathrm{NR}_{2}$, indicating the effective unzipping of MWCNTs. A new peak that appeared at $7^{\circ}$ corresponds to an interlayer distance of $1.26 \mathrm{~nm}$, and could be attributed to the catechol pyrrolidine groups and the introduction of oxygen-containing groups on the edge of each layer, similar to GO [36]. 


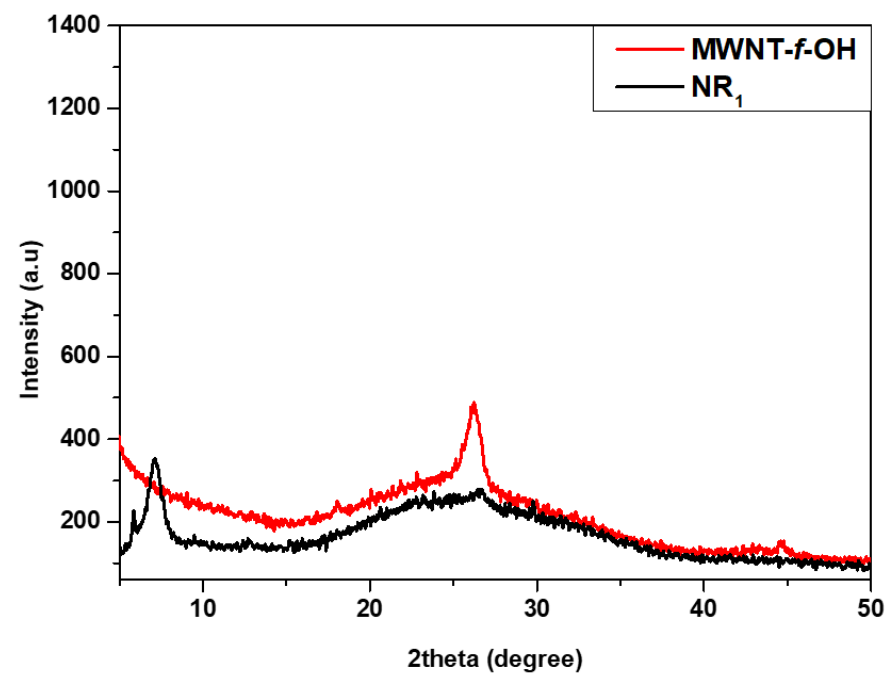

Figure 5. XRD diffraction pattern of MWCNT-f-OH and carbon nanoribbons $\mathrm{NR}_{2}$.

The Raman spectra of products $\mathrm{NR}_{1}$ and $\mathrm{NR}_{2}$ were like that of MWCNT-f-OH (see Figure 6). The characteristic D and G bands of MWCNT-f-OH and sample $\mathrm{NR}_{1}$ are located at 1359 and $1567 \mathrm{~cm}^{-1}$, respectively, where the same bands for the sample $\mathrm{NR}_{2}$ are blue shifted to 1350 and $1563 \mathrm{~cm}^{-1}$, respectively. The $2 \mathrm{D}$ band was located at $2724 \mathrm{~cm}^{-1}$ in the spectra of both the products and the MWCNT-f-OH. The relatively high value of the $\mathrm{I}_{\mathrm{D}} / \mathrm{I}_{\mathrm{G}}$ ratio of MWCNT-f-OH (0.77) is attributed to the added functional groups. Comparing the values for the $\mathrm{I}_{\mathrm{D}} / \mathrm{I}_{\mathrm{G}}$ ratio, which is 0.36 and 0.64 for the spectra of products $\mathrm{NR}_{2}$ and $\mathrm{NR}_{1}$, respectively, the much lower value recorded for $\mathrm{NR}_{2}$ reflects the increased ratio between the $\mathrm{sp}^{2}$ carbon atoms at the core and the $\mathrm{sp}^{3}$ carbon atoms at the edges, due to the much larger width of the $\mathrm{NR}_{2}$ product [22].

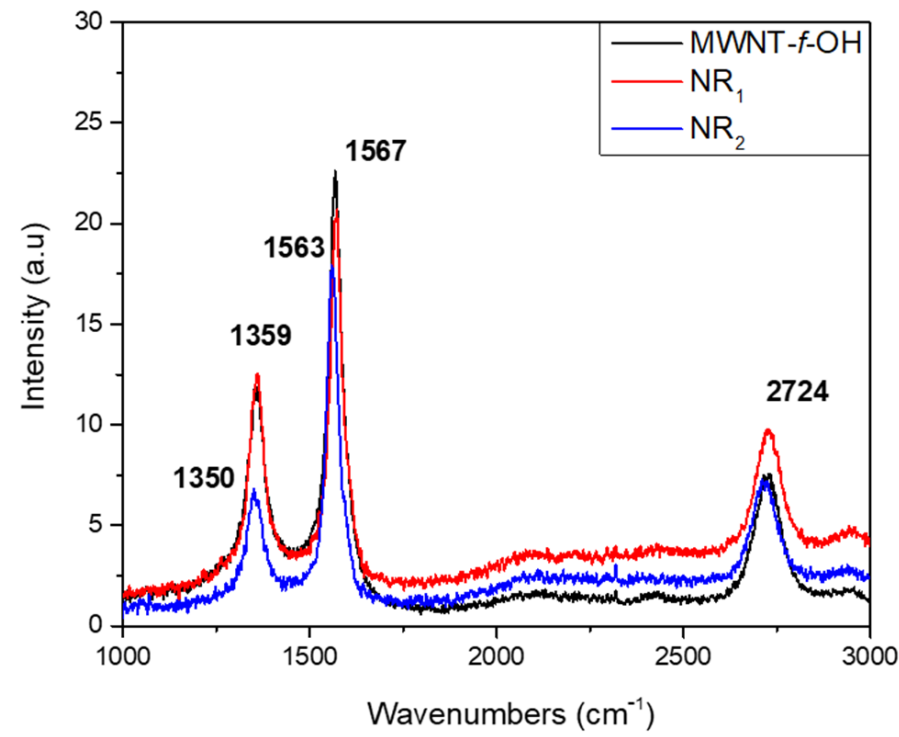

Figure 6. Raman spectra of MWCNT-f-OH and products $\mathrm{NR}_{1}$ and $\mathrm{NR}_{2}$ of carbon nanoribbons.

The XPS analysis of the sample $\mathrm{NR}_{2}$ showed the different functional chemical groups (see Figure 7). The main fitted peak at $284.6 \mathrm{eV}$ is due to the basic carbon frame created by $\mathrm{sp}^{2}$ and $\mathrm{sp}^{3}$ hybridization. A second intense peak at 285.9 is attributed to the C-OH groups, representing $23.1 \%$ of the whole carbon area. The remaining peaks are associated with the epoxy $(13.6 \%)$, carbonyl $(4.1 \%)$, and carboxyl (3.2\%) groups. The pi-pi* interactions may derive from the few layers nanoribbons while interacting together via aromatic systems. 

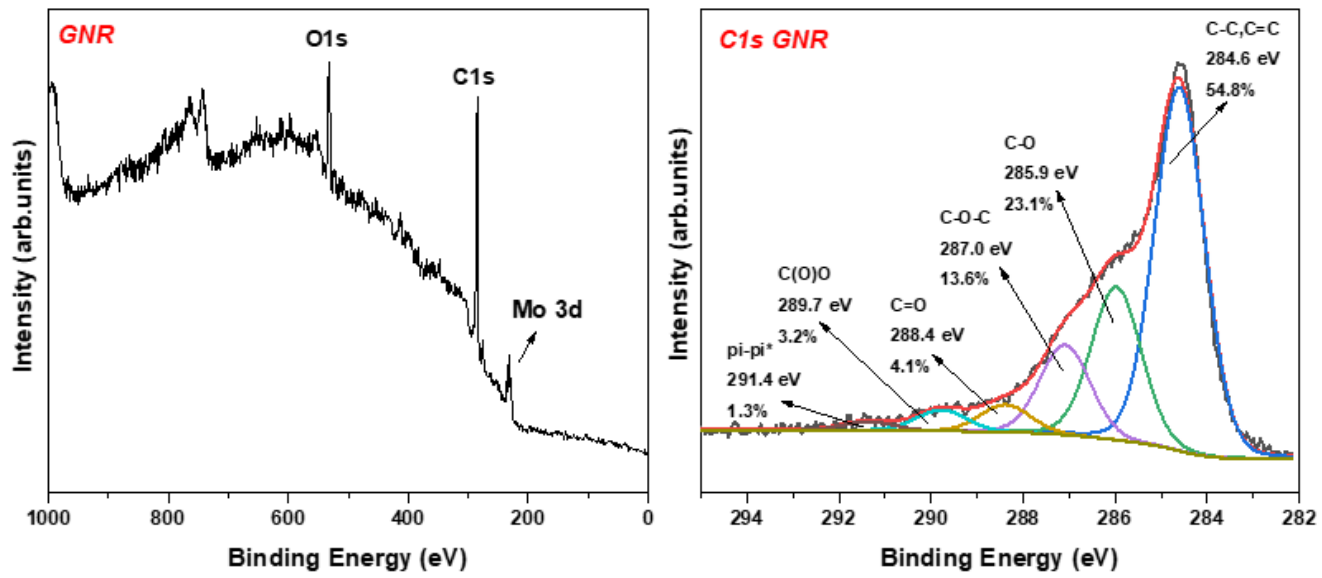

Figure 7. (Left) XPS survey of $\mathrm{NR}_{2}$, where the Mo peak is attributed to the substrate at which the sample was drop casted; (Right) C1s photoelectron peak of $\mathrm{NR}_{2}$.

The electrical conductivity of large nanoribbons $\mathrm{NR}_{2}$ was estimated to be $1075 \mathrm{~S} \mathrm{~m}^{-1}$ (sheet resistance: $186 \mathrm{Ohm} \mathrm{sq}^{-1}$ ) and increased to $1835 \mathrm{~S} \mathrm{~m}^{-1}$ (sheet resistance: $109 \mathrm{Ohm} \mathrm{sq}^{-1}$ ) after the reduction of the product with $\mathrm{NaBH}_{4}$. The conductivity of the nanoribbons was lower than that of the starting MWCNT-f-OH, which was measured to be $2940 \mathrm{~S} \mathrm{~m}^{-1}$ (sheet resistance: $68 \mathrm{Ohm} \mathrm{sq}^{-1}$ ); however, it was remarkably higher compared with similar nanoribbons in the literature and suitable for applications in nanoelectronics. In the literature, several procedures for the unzipping of MWCNTs have been described. Unfortunately, few reports presented a common description of the nanoribbon product, which usually contains the dimensions, dispersibility, conductivity or sheet resistance, yield, etc. In Table 1, the most important characteristics of some common methods for the preparation of carbon nanoribbons-mainly by unzipping MWNTs-are presented. The $\mathrm{KO}_{2}$-assisted hydrothermal treatment is one of the most eco-friendly, low-cost, and high-yield methods. Apparently, it is the only one that can produce simultaneously narrow nanoribbons with intense photoluminescence and wide nanoribbons with an electrical conductivity between the highest points, especially after reduction.

Table 1. Comparison of various method for the formation of nanoribbon

\begin{tabular}{cccccc}
\hline & Method & Width of Nanoribbons & Conductivity & Yield & Ref \\
\hline MWNT & $\mathrm{H}_{2} \mathrm{SO}_{4}-\mathrm{KMnO}_{4}$ & $>100 \mathrm{~nm}$ & poor & nearly $100 \%$ & {$[19]$} \\
MWNT & Air at $500{ }^{\circ} \mathrm{C} /$ sonication & $10-30 \mathrm{~nm}$ & good & $2 \%$ & {$[21]$} \\
Carbon black & $\mathrm{HNO}_{3} / 90^{\circ} \mathrm{C}$ & $30 \mathrm{~nm}$ & & $66 \%$ & {$[37]^{*}$} \\
MWNT & $\mathrm{KOH}^{\circ} 900^{\circ} \mathrm{C}$ & & $628 \mathrm{~S} \mathrm{~m}^{-1}$ & $53 \%$ & {$[38]$} \\
MWNT-f-OH & $\mathrm{KO}_{2} 12{ }^{\circ} \mathrm{C}$ hydrothermal & $30-150 \mathrm{~nm}$ & $1730 \mathrm{~S} \mathrm{~m}^{-1}$ & $>90 \%$ & $* *$ \\
\hline
\end{tabular}

* Nanoribbons with photoluminescence. ${ }^{* *}$ This work.

\subsection{Cutting of Graphene Nanosheets}

The same reaction was then applied to pristine graphene nanosheets. Graphene nanosheets were dispersed in water, and their hydrothermal treatment with $\mathrm{KO}_{2}$ was also performed successfully. The products of the reaction, after the removal of the unreacted material or the large graphene nanosheets, were also divided by the material that was dispersed in the supernatant and the precipitate. As revealed by TEM analysis (see Figure 8), the precipitate mainly consisted of large nanographenes (NG) with diameters between 50 and $100 \mathrm{~nm}$, and the supernatant created by graphene quantum dots (GQD) had a diameter that ranged between 2 and $6 \mathrm{~nm}$. GQDs were easily dispersible in water and had a characteristic photoluminescence, in contrast with the larger NGs, which are less dispersible in water and without photoluminescence. 


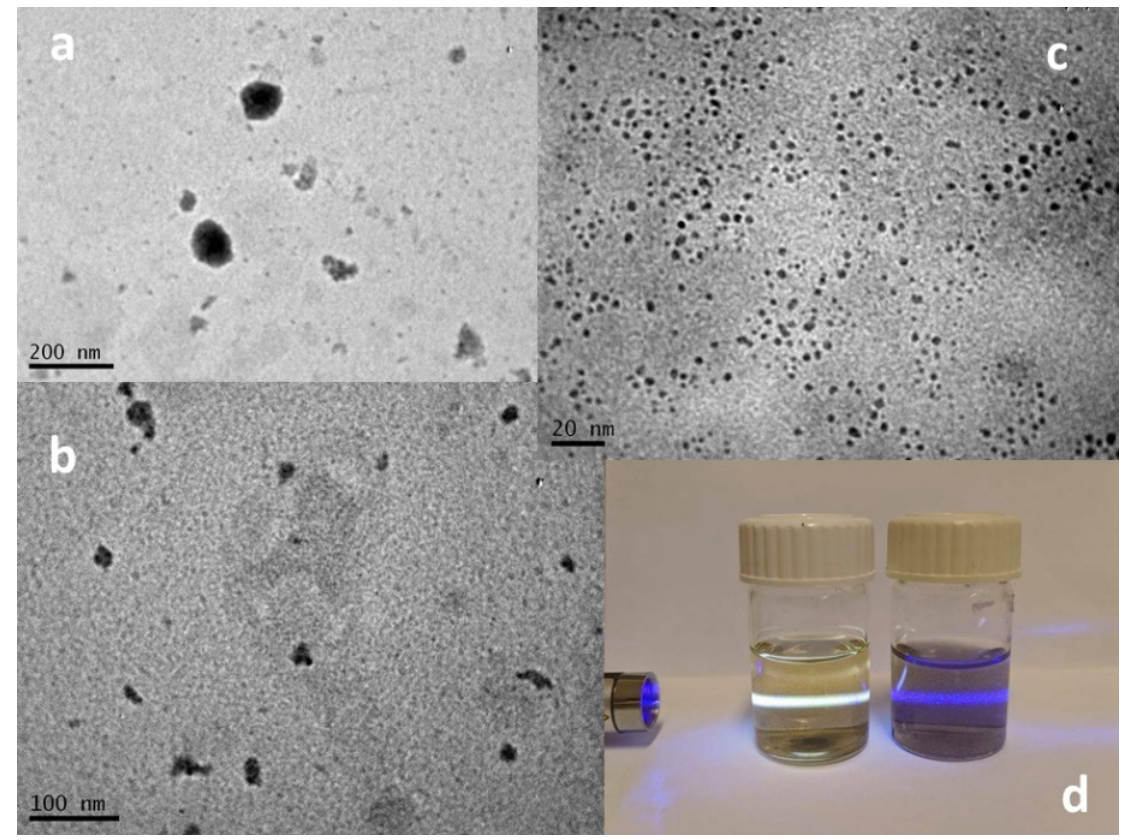

Figure 8. TEM images of (a,b) nanographenes (NGs) and (c) GQDs. (d) Photo of GQDs and NGs dispersed in water. GQDs emit white light after excitation with violet laser light, while NGs do not emit light.

The UV-Vis absorption spectrum of GQDs showed a wide band that starts from $800 \mathrm{~nm}$ and is continuously increased until the end of the spectrum measurement at $250 \mathrm{~nm}$. Two broad shoulders can be also observed at $280-300 \mathrm{~nm}$ (the $\pi, \pi^{*}$ transition) and between 450 and $550 \mathrm{~nm}$ (the $\mathrm{n}, \pi^{*}$ transition). The photoluminescence spectra of the GQD sample at several excitation wavelengths showed two different luminescence bands. The first with $\lambda_{\max }$ around $440 \mathrm{~nm}$ was recorded after excitation under 300 and $350 \mathrm{~nm}$, and the second was an excitation that depended on luminescence, with a maximum emission between 500 and $550 \mathrm{~nm}$ after excitation under 400 to $500 \mathrm{~nm}$ (see Figure 9). Interestingly, after the excitation with 300-350 nm, GNDs emitted a broad-band white light that extended from 400 to $600 \mathrm{~nm}$ because of the co-existence of the two different bands (see Figure $9 \mathrm{~b}$ ). The different emission bands here could be related to the size of the GNDs. According to Ajayan et al., the energy gap of the GNDs decreases with the increasing size [39]. Thus, the broad emission band is a result of the co-existence of GNDs with sizes between 2 and $6 \mathrm{~nm}$, as revealed also by the TEM image (see Figure 8c).

The Raman spectra of the two products showed the characteristic peaks of the D, G, and $2 \mathrm{D}$ bands at 1360,1570 , and $2737 \mathrm{~cm}^{-1}$, respectively. Both products have an increased $\mathrm{I}_{\mathrm{D}} / \mathrm{I}_{\mathrm{G}}$ ratio compared to that of pristine graphene nanosheets, due to the increased number of $\mathrm{sp}^{3}$ carbon atoms at the edges of the much smaller GQDs and NGs. The difference between the $I_{D} / I_{G}$ ratio of the two products, as shown in Figure 10, can be reasonably attributed to the fact that NGs have more $\mathrm{sp}^{3}$ carbon atoms due to the existence of defects at the core apart from the edges.

The XPS analysis of nanographene showed different types of oxygen functional groups (see Figure 11). The main peak at $284.6 \mathrm{eV}$ is due to $\mathrm{C}-\mathrm{C}$ and $\mathrm{C}=\mathrm{C}$ bonds, while the peak at 286.1 is ascribed to the $\mathrm{C}-\mathrm{OH}$ bonds, representing $18.3 \%$ of the carbon area. The less intense peaks at $287.2 \mathrm{eV}(7.0 \%), 288.2 \mathrm{eV}(7.0 \%)$, and $289.6 \mathrm{eV}(2.8 \%)$ represent the $\mathrm{C}-\mathrm{O}-\mathrm{C}$, $\mathrm{C}=\mathrm{O}$, and $\mathrm{COOH}$ groups, respectively. Finally, the contribution of $\mathrm{pi}^{-\mathrm{pi}^{*} \text { interactions }}$ was observed; this is characteristic for carbon in aromatic compounds between $\pi$ electron systems, such as aromatic rings. 

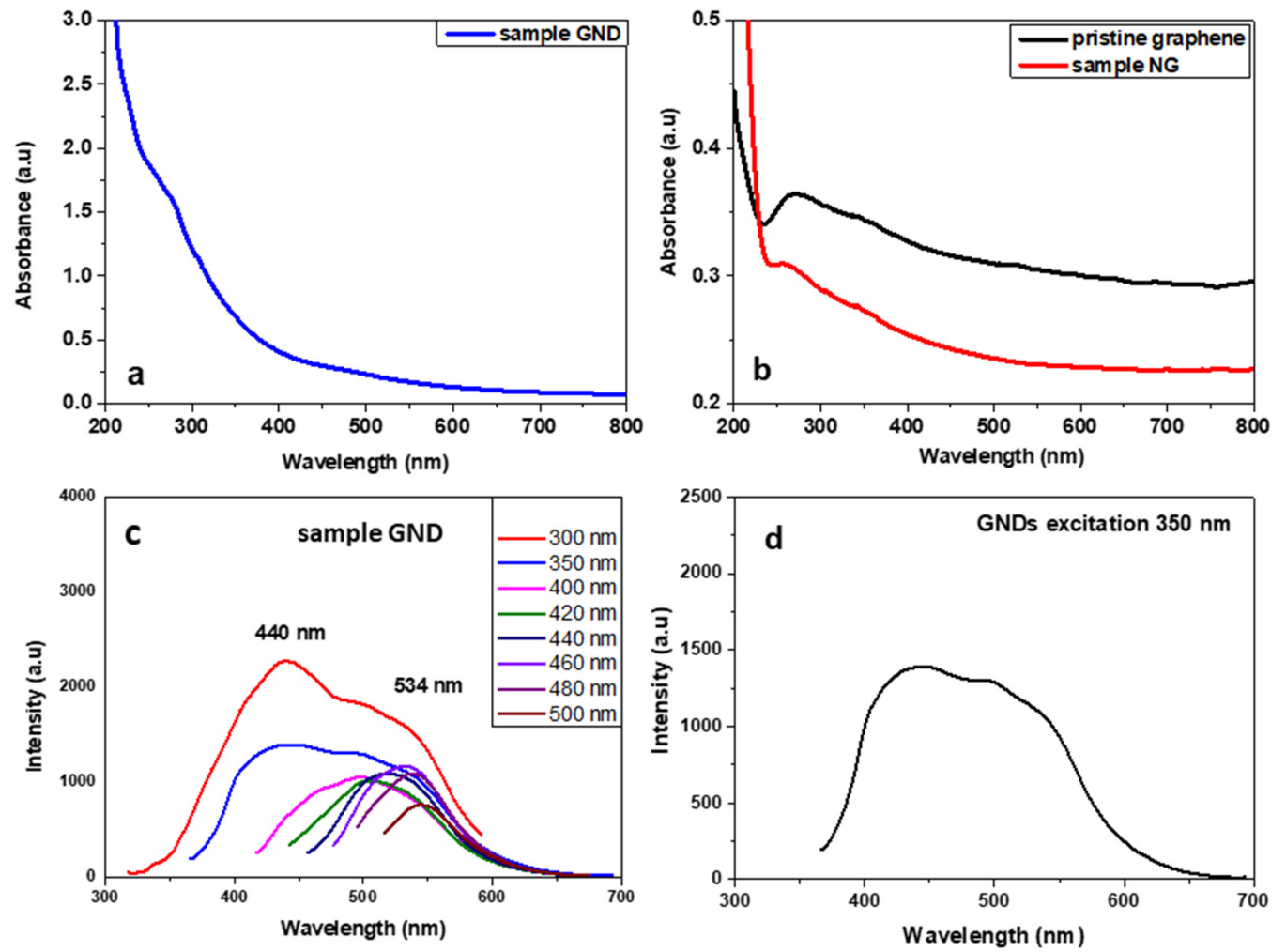

Figure 9. UV-Vis spectra of product GND (a), product NGs and pristine graphene (b), and PL spectra of GQD sample after excitation at several wavelengths (c). Broadband emission after excitation of GNDs with $350 \mathrm{~nm}(\mathbf{d})$.
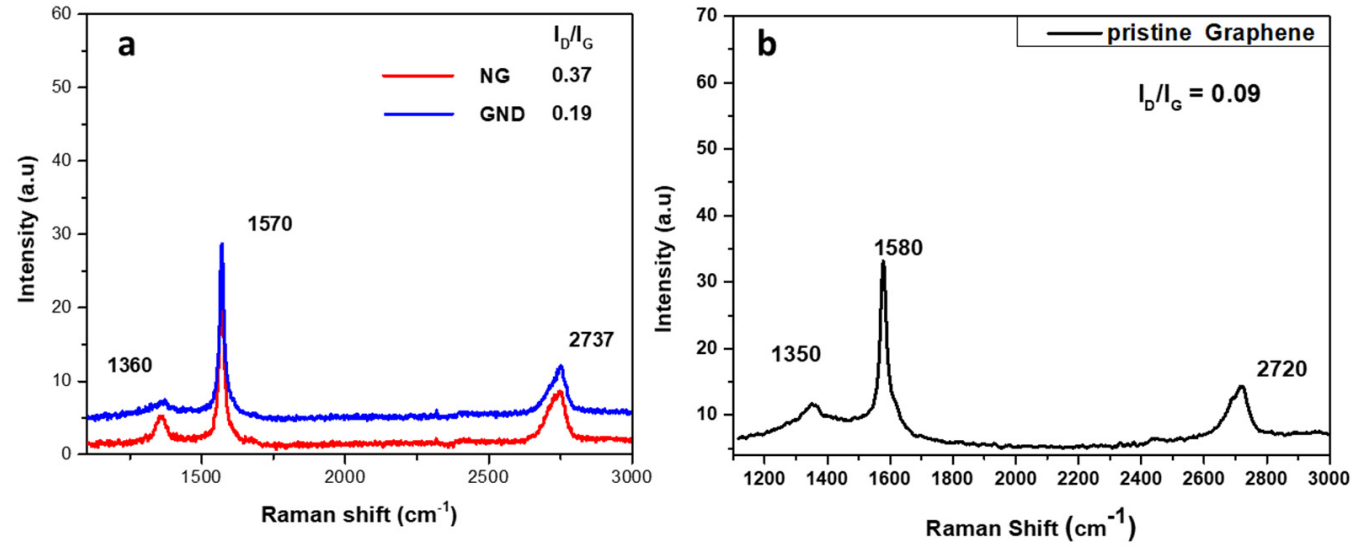

Figure 10. Raman spectra of (a) NG and GQDs; (b) pristine graphene.

The effectiveness of the $\mathrm{KO}_{2}$-assisted hydrothermal treatment for both substrates was indicated by the relatively high yield from unzipping MWCNT-f-OH or cutting graphene nanosheets. For the first procedure, the yield was above $90 \%$, as indicated by the limited number of unreacted MWCNT-f-OH that was observed in the TEM images. As regards the hydrothermal cutting of graphene, the $\mathrm{KO}_{2}$ assistance resulted in a remarkable yield that reached $50 \%$. The $\mathrm{KO}_{2}$-assisted hydrothermal treatment has proven to be an effective top-down method for the preparation of nanosized carbon structures from different graphenic precursors, such as carbon nanotubes, pristine graphene, or graphene oxide [34]. Preliminary results showed that carbon black could be also a precursor. Although the emissive NR1 and GQDs are very interesting nanomaterials, which potentially could be used in optoelectronic applications, the main products here are the wide, highly conductive nanoribbons $\mathrm{NR}_{2}$ and nanographenes NG (50-100 nm). The first could be used for the 
preparation of transparent conductive films in several applications, such as photovoltaics, smart screens, etc. Finally, nanographenes seems to have the suitable size to be used in bioapplications, such as drug delivery, biosensing, etc.
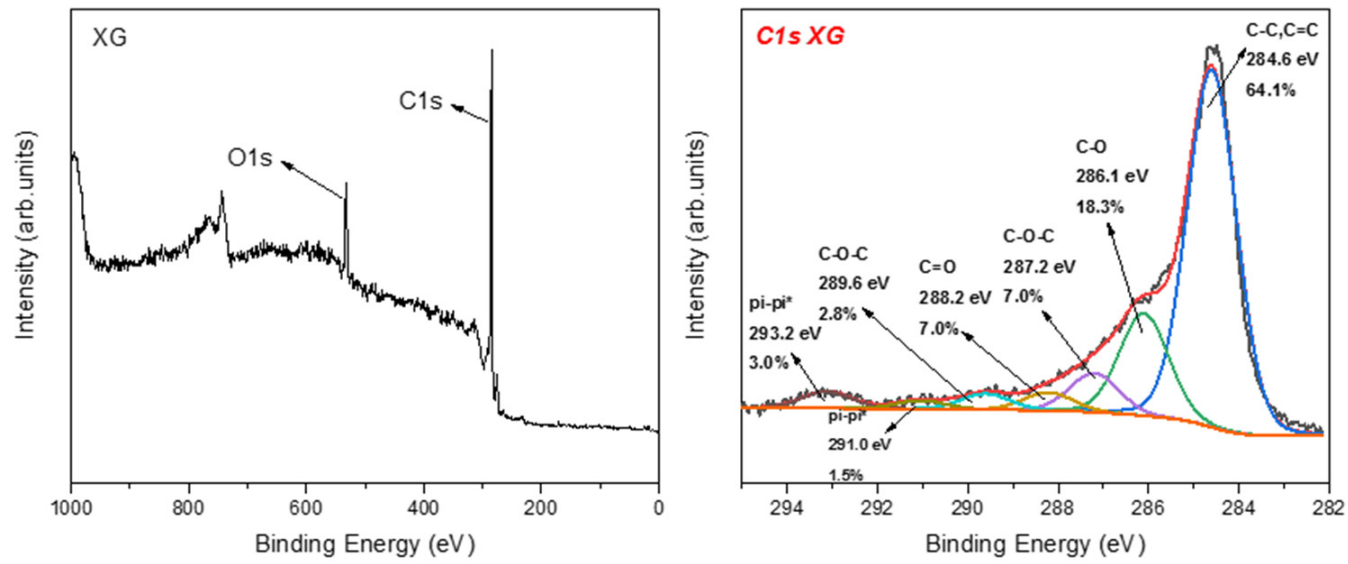

Figure 11. (Left) XPS survey of NG and (right) C1s photoelectron peak of Ng.

The mechanism. The mechanism of this effective cutting of graphenic layers or unzipping carbon nanotubes has been already proposed by Zhao et al., who showed that $\mathrm{KO}_{2}$ hydrothermally cut GO into very small pieces, thus producing GQDs with yellow PL emission [34]. It is known that $\mathrm{KO}_{2}$ in water produces an intermediate superoxide anion $\mathrm{O}_{2}{ }^{-}$, and finally $\mathrm{OH}^{-}$and $\mathrm{O}_{2}$. The mechanism proposed by $\mathrm{Zhao}$ et al. is based on the existence of epoxy groups along lines on the GO surface or the formation of new lines of epoxy groups by the strongly oxidative superoxide anion $\mathrm{O}_{2}{ }^{-}$, and the dissociation of the underlying $\mathrm{C}-\mathrm{C}$ bonds by $\mathrm{OH}^{-}$during the hydrothermal heating. In our work, functionalized MWCNTs and pristine graphene-without epoxy groups-were used, which means that epoxy lines should be formed exclusively during the $\mathrm{KO}_{2}$ treatment of the starting material (see Figure 12).
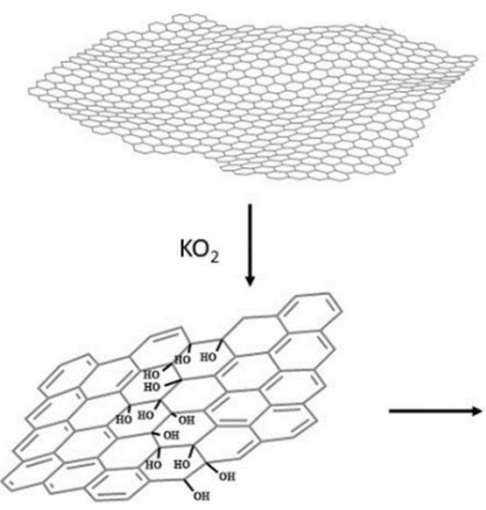
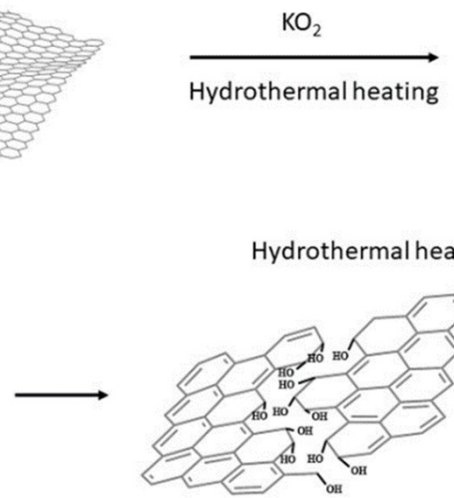
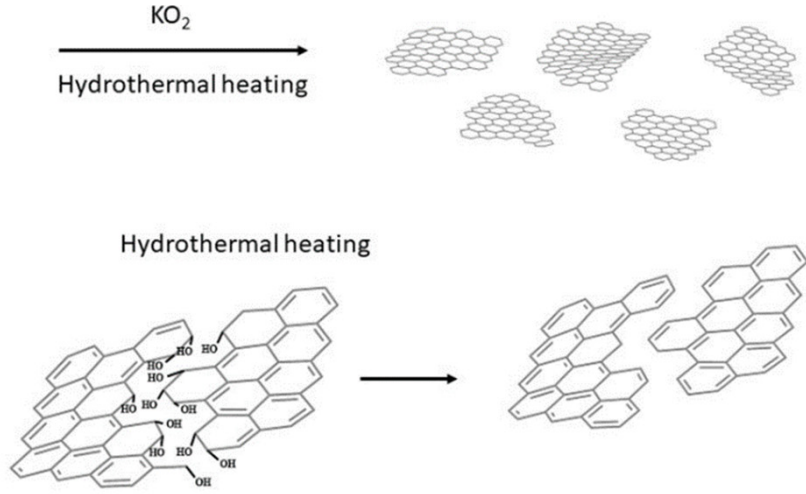

Figure 12. The proposed mechanism of the cutting of graphene nanosheets.

To support this hypothesis, pristine graphene and MWCNT-f-OH were mixed with an excess of $\mathrm{KO}_{2}$ in water under sonication for a few minutes, and the product was separated and washed repeatedly to remove by-products. The $\mathrm{KO}_{2}$-treated graphene and MWCNT-f$\mathrm{OH}$ were characterized by FTIR spectroscopy, which showed in both cases the formation of a strong band at $1350 \mathrm{~cm}^{-1}$ and a broad band at $3150-3200 \mathrm{~cm}^{-1}$ (see Figure 13). 

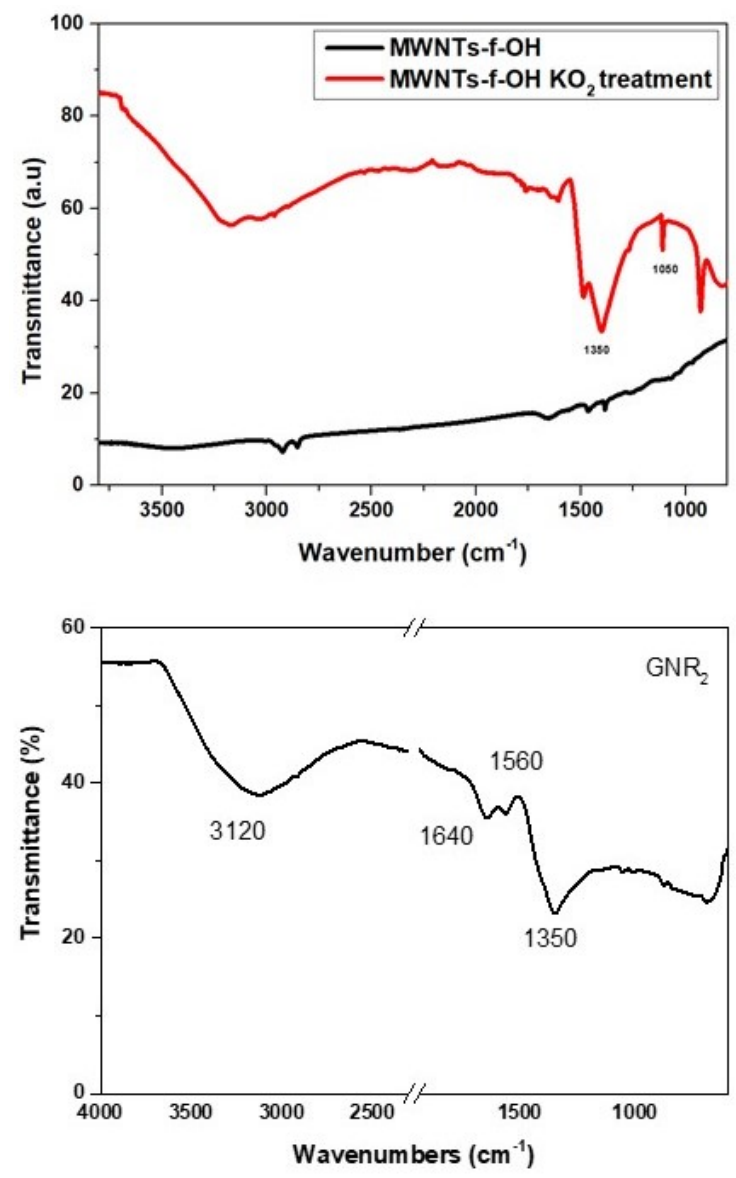

Figure 13. IR spectra of the graphene or MWCNT-f-OH after the treatment with $\mathrm{KO}_{2}$ in water.

The latter can be attributed to $\mathrm{OH}$ stretching vibrations and the former to $\mathrm{C}-\mathrm{O}$ bending vibrations or epoxy groups. The lack of any other characteristic peak in the FT-IR spectra of both samples indicates the formation of epoxy groups, which probably was partly hydrolyzed to $\mathrm{C}-\mathrm{OH}$ during the $\mathrm{KO}_{2}$ treatment of graphene or MWCNT-f-OH, and before the hydrothermal heating in the presence of $\mathrm{OH}^{-}$, which favors the cutting or unzipping of the epoxy or hydroxy functionalized graphene or MWCNT-f-OH surface.

\section{Conclusions}

The hydrothermal treatment of hydrophilic-functionalized MWCNTs with $\mathrm{KO}_{2}$ leads to the effective unzipping and production of GNR. The same reaction was successfully applied to pristine graphene, creating nanographenes and GQDs. In both cases, there is a hydrophilic emissive nanostructure that was isolated by the water phase-narrow GNRs or GQDs-and a less hydrophilic, non-emissive part that isolated a precipitate that contains larger GNRs or nanographenes. Interestingly, narrow GNRs and GQDs are emissive nanostructures and, significantly, GQDs emit white light after excitation at $350 \mathrm{~nm}$.

Supplementary Materials: The following are available online at https:/ /www.mdpi.com/article/10.3 390/nano12030447/s1, Figure S1: AFM images of GNR from sample NR 1 ; Figure S2. AFM images of GNR from the sample $\mathrm{NR}_{2}$.

Author Contributions: Conceptualization, A.K. and V.G.; methodology, K.S., N.C. and A.K.; writingoriginal draft preparation, K.S., N.C., A.K. and V.G.; writing-review and editing, V.G. and D.G.; supervision, V.G. and D.G. All authors have read and agreed to the published version of the manuscript.

Funding: This research received no external funding.

Data Availability Statement: Not applicable. 
Acknowledgments: The authors gratefully acknowledge M. Kollia and the Laboratory of Electron Microscopy and Microanalysis, School of Natural Sciences, University of Patras for the TEM measurements.

Conflicts of Interest: The authors declare no conflict of interest.

\section{References}

1. Georgakilas, V.; Perman, J.A.; Tucek, J.; Zboril, R. Broad family of carbon nanoallotropes: Classification, chemistry, and applications of fullerenes, carbon dots, nanotubes, graphene, nanodiamonds, and combined superstructures. Chem. Rev. 2015, 115, 4744-4822. [CrossRef]

2. Kim, S.D.; Sarkar, A.; Ahn, J.H. Graphene-based nanomaterials for flexible and stretchable batteries. Small 2021, 17, 2006262. [CrossRef]

3. Anichini, C.; Samorì, P. Graphene-based hybrid functional materials. Small 2021, 17, 2100514. [CrossRef]

4. Zhu, J.; Yang, D.; Yin, Z.; Yan, Q.; Zhang, H. Graphene and graphene-based materials for energy storage applications. Small 2014, 10, 3480-3498. [CrossRef]

5. Yu, L.P.; Shearer, C.; Shapter, J. Recent development of carbon nanotube transparent conductive films. Chem. Rev. 2016, 116, 13413-13453. [CrossRef]

6. Clancy, A.J.; Bayazit, M.K.; Hodge, S.A.; Skipper, N.T.; Howard, C.A.; Shaffer, M.S.P. Charged carbon nanomaterials: Redox chemistries of fullerenes, carbon nanotubes, and graphenes. Chem. Rev. 2018, 118, 7363-7408. [CrossRef]

7. Schroeder, V.; Savagatrup, S.; He, M.; Lin, S.; Swager, T.M. Carbon nanotube chemical sensors. Chem. Rev. 2019, 119, 599-663. [CrossRef]

8. Peng, Z.; Zhao, T.; Zhou, Y.; Li, S.; Li, J.; Leblanc, R.M. Bone tissue engineering via carbon-based nanomaterials. Adv. Healthc. Mater. 2020, 9, 1901495. [CrossRef]

9. Feng, L.; $\mathrm{Wu}, \mathrm{L}$; $\mathrm{Qu}, \mathrm{X}$. New horizons for diagnostics and therapeutic applications of graphene and graphene oxide. Adv. Mater. 2012, 25, 168-186. [CrossRef]

10. Song, S.; Shen, H.; Wang, Y.; Chu, X.; Xie, J.; Zhou, N.; Shen, J. Biomedical application of graphene: From drug delivery, tumor therapy, to theranostics. Colloids Surf. B Biointerfaces 2020, 185, 110596. [CrossRef]

11. Chen, H.-J.; Zhang, Z.-H.; Xie, D.; Cai, R.; Chen, X.; Liu, Y.-N.; Yao, S.-Z. Surface-imprinting sensor based on carbon nanotubes/graphene composite for determination of bovine serum albumin. Electroanalysis 2012, 24, 2109-2116. [CrossRef]

12. Tang, F.; Gao, J.; Ruan, Q.; Wu, X.; Wu, X.; Zhang, T.; Liu, Z.; Xiang, Y.; He, Z.; Wu, X. Graphene-wrapped MnO/C composites by MOFs-derived as cathode material for aqueous zinc ion batteries. Electrochim. Acta 2020, 353, 136570. [CrossRef]

13. Mendes, R.G.; Mandarino, A.; Koch, B.; Meyer, A.K.; Bachmatiuk, A.; Hirsch, C.; Gemming, T.; Schmidt, O.G.; Liu, Z.; Rümmeli, M.H. Size and time dependent internalization of label-free nano-graphene oxide in human macrophages. Nano Res. 2017, 10, 1980-1995. [CrossRef]

14. Kim, J.; Kim, S.W.; Yun, H.; Kim, B.J. Impact of size control of graphene oxide nanosheets for enhancing electrical and mechanical properties of carbon nanotube-polymer composites. RSC Adv. 2017, 7, 30221-30228. [CrossRef]

15. Wang, H.; Wang, H.S.; Ma, C.; Chen, L.; Jiang, C.; Chen, C.; Xie, X.; Li, A.-P.; Wang, X. Graphene nanoribbons for quantum electronics. Nat. Rev. Phys. 2021, 3, 791-802. [CrossRef]

16. Johnson, A.P.; Gangadharappa, H.V.; Pramod, K. Graphene nanoribbons: A promising nanomaterial for biomedical applications. J. Control. Release 2020, 325, 141-162. [CrossRef]

17. Zakharova, O.V.; Mastalygina, E.E.; Golokhvast, K.S.; Gusev, A.A. Graphene nanoribbons: Prospects of application in biomedicine and toxicity. Nanomaterials 2021, 11, 2425. [CrossRef]

18. Chen, Z.; Narita, A.; Müllen, K. Graphene nanoribbons: On-surface synthesis and integration into electronic devices. Adv. Mater. 2020, 32, 2091893. [CrossRef]

19. Kosynkin, D.V.; Higginbotham, A.L.; Sinitskii, A.; Lomeda, J.R.; Dimiev, A.B.; Price, K.; Tour, J.M. Longitudinal unzipping of carbon nanotubes to form graphene nanoribbons. Nature 2009, 458, 872-876. [CrossRef]

20. Higginbotham, A.L.; Kosynkin, D.V.; Sinitskii, A.; Sun, Z.; Tour, J.M. Lower-defect graphene oxide nanoribbons from multiwalled carbon nanotubes. ACS Nano 2010, 4, 2059-2069. [CrossRef]

21. Jiao, L.; Wang, X.; Diankov, G.; Wang, H.; Dai, H. Facile synthesis of high-quality graphene nanoribbons. Nat. Nanotechnol. 2010, 5, 321-325. [CrossRef] [PubMed]

22. Castillo-Martinez, E.; Carretero-Gonzalez, J.; Sovicha, J.; Lima, M.D. High temperature structural transformations of few layer graphene nanoribbons obtained by unzipping carbon nanotubes. J. Mater. Chem. A 2014, 2, 221. [CrossRef]

23. Mondal, S.; Ghosh, S.; Raj, C.R. Unzipping of single-walled carbon nanotube for the development of electrocatalytically active hybrid catalyst of graphitic carbon and pd nanoparticles. ACS Omega 2018, 3, 622-630. [CrossRef] [PubMed]

24. Vo, T.H.; Shekhirev, M.; Kunkel, D.A.; Morton, M.D.; Berglund, E.; Kong, L.; Wilson, P.M.; Dowben, P.; Enders, A.; Sinitskii, A. Large-scale solution synthesis of narrow graphene nanoribbons. Nat. Commun. 2014, 5, 3189. [CrossRef]

25. Zhao, S.; Rondin, L.; Delport, G.; Voisin, C.; Beser, U.; Hu, Y.; Feng, X.; Müllen, K.; Narita, A.; Campidelli, S.; et al. Fluorescence from graphene nanoribbons of well-defined structure. Carbon 2017, 119, 235-240. [CrossRef]

26. Li, G.; Yoon, K.Y.; Zhong, X.; Zhu, X.; Dong, G. Efficient bottom-up preparation of graphene nanoribbons by mild Suzuki-Miyaura polymerization of simple triaryl monomers. Chem. Eur. J. 2016, 22, 9116-9120. [CrossRef] 
27. Denk, R.; Hohage, M.; Zeppenfeld, P.; Cai, J.; Pignedoli, C.A.; Sode, H.; Fasel, R.; Feng, X.; Mullen, K.; Wang, S.; et al. Excitondominated optical response of ultra-narrow graphene nanoribbons. Nat. Commun. 2014, 5, 4253. [CrossRef]

28. Xie, M.; Su, Y.; Lu, X.; Zhang, Y.; Yang, Z.; Zhang, Y. Blue and green photoluminescence graphene quantum dots synthesized from carbon fibers. Mater. Lett. 2013, 93, 161-164. [CrossRef]

29. Xue, Q.; Huang, H.; Wang, L.; Chen, Z.; Wu, M.; Li, Z.; Pan, D. Nearly monodisperse graphene quantum dots fabricated by amine-assisted cutting and ultrafiltration. Nanoscale 2013, 5, 12098-12103. [CrossRef]

30. Zhang, M.; Bai, L.; Shang, W.; Xie, W.; Ma, H.; Fu, Y.; Fang, D.; Sun, H.; Fan, L.; Han, M.; et al. Facile synthesis of water-soluble, highly fluorescent graphene quantum dots as a robust biological label for stem cells. J. Mater. Chem. 2012, 22, 7461. [CrossRef]

31. Pan, D.; Zhang, J.; Li, Z.; Wu, M. Hydrothermal route for cutting graphene sheets into blue-luminescent graphene quantum dots. Adv. Mater. 2010, 22, 734-738. [CrossRef]

32. Yang, F.; Zhao, M.; Zheng, B.; Xiao, D.; Wu, L.; Guo, Y. Influence of pH on the fluorescence properties of graphene quantum dots using ozonation pre-oxide hydrothermal synthesis. J. Mater. Chem. 2012, 22, 25471. [CrossRef]

33. Shen, J.; Zhu, Y.; Yang, X.; Zong, J.; Zhang, J.; Li, C. One-pot hydrothermal synthesis of graphene quantum dots surface-passivated by polyethylene glycol and their photoelectric conversion under near-infrared light. New J. Chem. 2012, 36, 97-101. [CrossRef]

34. Zhao, Y.; Wu, X.; Sun, S.; Ma, L.; Zhang, L.; Lin, H. A facile and high-efficient approach to yellow emissive graphene quantum dots from graphene oxide. Carbon 2017, 124, 342-347. [CrossRef]

35. Koutsioukis, A.; Belessi, V.; Georgakilas, V. Solid phase functionalization of MWNTs: An eco-friendly approach for carbon-based conductive ink. Green Chem. 2021, 23, 5442-5448. [CrossRef]

36. Belessi, V.; Petridis, D.; Steriotis, T.; Spyrou, K.; Manolis, G.K.; Psycharis, V.; Georgakilas, V. Simultaneous reduction and surface functionalization of graphene oxide for highly conductive and water dispersible graphene derivatives. SN Appl. Sci. $2019,1,77$. [CrossRef]

37. Khodabakhshi, S.; Fulvio, F.P.; Sousaraei, A.; Kiani, S.; Niu, Y.; Palmer, R.E.; Kuo, C.H.W.; Rudd, J.; Barron, R.A.; Andreoli, E. Oxidative synthesis of yellow photoluminescent carbon nanoribbons from carbon black. Carbon 2021, 183, 495-503. [CrossRef]

38. Han, J.; Kim, W.; Kim, H.K.; Youn, H.C.; Han, J.T.; Kim, W.; Roh, K.C. Longitudinal unzipped carbon nanotubes with high specific surface area and trimodal pore structure. RSC Adv. 2016, 6, 8661. [CrossRef]

39. Peng, J.; Gao, W.; Gupta, B.K.; Liu, Z.; Romero-Aburto, R.; Ge, L.; Song, L.; Alemany, L.B.; Zhan, X.; Gao, G.; et al. Graphene quantum dots derived from carbon fibers. Nano Lett. 2012, 12, 844-849. [CrossRef] 